\title{
Self-Heating Effects on the Thermal Noise of Deep Sub-Micron FD-SOI MOSFETs
}

\author{
Can Baltac1, Yusuf Leblebici \\ Microelectronic Systems Laboratory \\ École Polytechnique Fédérale de Lausanne \\ Email: can.baltaci@epfl.ch,yusuf.leblebici@epfl.ch
}

\begin{abstract}
Self-heating effects became more prominent with the introduction of the modern devices like FD-SOI and low thermal conductivity materials such as $\mathrm{SiO}_{2}$. Consequently, the temperature rise of a device due to its self-heating is pronounced more as the gate lengths shrink and the power density values increase. In analog design, one of the main drawbacks of elevated temperature is the deterioration of the thermal noise performance. For observing the thermal noise performance of FD-SOI MOSFETs, a thermal model for the device self-heating is used. The influence of self-heating on the thermal noise is examined by activating and inactivating the self-heating thermal model and comparing the results. It is shown that self-heating can deteriorate the thermal noise current (up to $18 \%$ ) and the input referred thermal noise voltage (up to 37\%) significantly for short channel FD-SOI devices.
\end{abstract}

Keywords-Self-heating, FD-SOI, thermal noise, thermal modelling

\section{INTRODUCTION}

One of the main drawbacks of device scaling is the increased power density and the resulting elevated temperature. Operation at high temperature has many drawbacks like leakage, lower mobility, electro-migration and so on [1]. One of the main drawbacks is the increased device thermal noise in analog design since thermal noise has a direct relation with the absolute local temperature of the device. Especially after the introduction of the modern MOSFET device geometries like Fully Depleted Silicon on Insulator (FD-SOI), self-heating became a more critical problem [2]. This is mainly due to the low thermal conductivity materials like $\mathrm{SiO}_{2}$ which are necessary for the implementation of FD-SOI devices [3]-[5]. In these devices, low thermal conductivity barriers surround the channel, in which the power is mostly dissipated. Consequently, the generated heat in FD-SOI MOSFETs cannot find a lowresistance path for diffusing outside the device borders. The generated heat turns into elevated temperature in nanometer scale local spots, which are comparable to the dimensions of transistors in FD-SOI. The device temperature becomes even higher when compared to the average temperature of the chip, which is already much larger than the room temperature due to the high-density power dissipation of the other neighbour devices. Therefore, it is necessary to take into account the temperature rise due to the self-heating of the device while designing a block where low noise operation is critical.

This work is intended to analyse the thermal noise of a device considering its self-heating and show the influence of self-heating on its thermal noise performance. For that, a selfheating thermal model is used for activating and inactivating the self-heating effects and observing the significance of the elevated temperature due to device self-heating. The thermal model for the self-heating is included in the device model by the design kit, which is a commercially available 28nm FDSOI technology.

In Section II, the theory of thermal noise in MOSFET devices is given in addition to different short channel thermal noise models proposed by different authors. In Section III, thermal noise current and input referred thermal noise voltage performance of FD-SOI MOSFET devices are compared for the cases where self-heating is activated and inactivated. Finally, in Section IV, the summary of the work and the conclusions are provided.

\section{TheORETICAL BACKGROUND AND RELATED WORK}

The power spectral density of the thermal noise current in the channel of a MOSFET device is given by [6]

$$
S_{i}=4 k T \gamma g_{d 0}
$$

where $k$ is the Boltzmann constant, $T$ is the absolute temperature, $g_{d 0}$ is the zero-bias drain conductance of the MOSFET device (i.e. the drain conductance of the device when $\mathrm{V}_{\mathrm{DS}}=0 \mathrm{~V}$ is applied) and $\gamma$ is the excess noise coefficient. For long channel devices in strong inversion, $\gamma$ takes values between 1 and $2 / 3$ while moving from linear region to saturation. However, for devices with a short channel, the value of $\gamma$ tends to be larger due do various factors. Channel length modulation is one of the effects that increase $\gamma$ for short channel devices. Increasing $\mathrm{V}_{\mathrm{DS}}$ creates a pinch-off region on the drain end and decreases the effective length of the device where the inversion layer charge is present in the channel. This would increase the thermal noise current as the device moves deeper into saturation [7]. Velocity saturation is another factor that affect the charge density and consequently the thermal noise current of the device. In [8]-[10] the authors included velocity saturation in the derivation of the thermal noise current and observations made on an increasing trend of $\gamma$ under smaller channel lengths and higher velocity saturation.

Most of the authors explain the large $\gamma$ by hot electron effect [8], [10]-[12]. This effect is mainly observed in short channels under large $\mathrm{V}_{\mathrm{DS}}$ voltage. Large lateral electric field in the 
channel creates hot electrons which have higher temperature (i.e. larger average kinetic energy) when compared to the lattice temperature. In that case, the average temperature of these hot electrons has to be considered rather than the lattice temperature. The temperature of the hot electrons can be approximated by [13]

$$
T_{e}=T_{L}\left(1+\frac{E}{E_{c}}\right)^{n}
$$

where $T_{e}$ is the hot electron temperature, $T_{L}$ is the lattice temperature, $E_{c}$ is the critical electric field and $E$ is the lateral electric field in the channel with $n=2,1$, or 0 . While $n=$ 0 corresponds to no hot carriers case, $n=2$ gives the other extreme. In [10], (2) is modified as $T_{e}=T_{L}+\delta T_{L}\left(\frac{E}{E_{c}}\right)^{2}$ where $\delta$ is used as a fitting parameter to adjust the simulations to the measurement. With the hot carrier model, the higher temperature electrons show more random fluctuations and this can be modelled by providing a larger $\gamma$ in (1).

Another explanation of larger $\gamma$ under very large $\mathrm{V}_{\mathrm{DS}}$ is the avalanche multiplication [11], [14]. When $\mathrm{V}_{\mathrm{DS}}$ is larger than the band-gap voltage, high energy carriers are scattered by valence band electrons and additional electron-hole pairs are created. The thermal fluctuations of these additional carriers contribute to the overall noise and this increases $\gamma$.

The listed modifications on the thermal noise current provide good approximations for the short channel devices; however, they don't account for the self-heating of individual devices for a large range of bias conditions or they assume the increase in $\gamma$ due to self-heating is already included in the model by fitting between measurements and simulations. For obtaining more realistic values for the power spectral density of thermal noise current under different bias conditions, one has to estimate the individual temperature of a device accounting its self-heating effects. Most of the current simulators can find solutions under a single temperature input that is valid for all the devices in the net-list. Consequently, different devices with different lattice temperatures are assumed to have the same temperature value. This would result in some errors in the calculation of the thermal noise current and hence in the noise figure of amplifiers, especially when they are implemented in advanced technologies like FD-SOI, FinFET where the devices are confined inside low thermal conductance materials. As it has been already mentioned, very large temperature gradients and different temperature values in short distances can be observed in these technologies, mainly due to low thermal conductance and high power densities [2]-[5]. Therefore, the noise analysis should be performed simultaneously by considering the self-heating effects.

\section{Thermal Noise Considering Self-Heating}

\section{A. Thermal Model for Self-Heating}

The temperature rise of each device due to their individual self-heating is calculated by considering the thermal model of Fig. 1. According to the model, $R_{t h}$ is the thermal resistance and $C_{t h}$ is the heat capacitance. The values of these

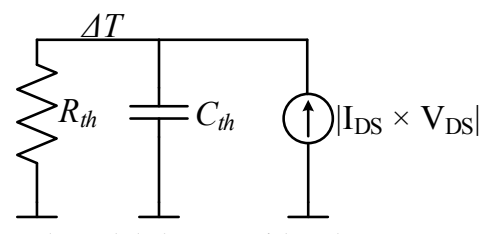

Fig. 1: The thermal model that provides the temperature rise of each device due to their self-heating [15].

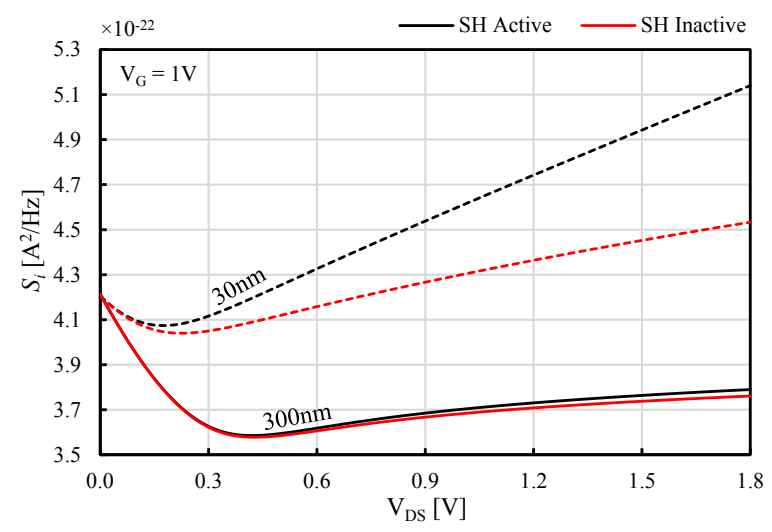

Fig. 2: Thermal noise current $\left(S_{i}\right)$ of $30 \mathrm{~nm}$ and $300 \mathrm{~nm}$ nMOS devices for the cases where self-heating thermal model is active and inactive.

parameters are calculated for each device according to their geometries (width, length, number of fingers etc.) The power dissipation of the device is modelled by a current source. The temperature value on the upper common node gives the temperature increase $\Delta T$ of the device due to its selfheating. The complete model is included in the device by the design kit provider and can be switched on or off for a specific simulation. Consequently, with the provided selfheating model, it is possible to consider the self-heating effects for all bias conditions.

\section{B. Thermal Noise Current}

For observing the effect of self-heating on the thermal noise current, nMOS devices with different gate lengths are simulated by activating and inactivating the self-heating thermal model. The gate lengths of the devices are varied from $30 \mathrm{~nm}$ to $300 \mathrm{~nm}$. The gate voltage is kept constant and $V_{D S}$ is set as the sweep parameter. All devices are implemented with 20 fingers and their finger width is adjusted so that their zero bias drain conductance is set approximately equal to $20 \mathrm{~mA} / \mathrm{V}$. Like that, all devices have equal thermal noise current under no power dissipation and no self-heating. Fig. 2 shows the thermal noise current. The relatively large thermal noise current in the 0 $300 \mathrm{mV}$ window is due to linear region operation. The increase in the noise with $\mathrm{V}_{\mathrm{DS}}$ in the saturation can be explained with velocity saturation and hot electrons. In addition to these observations, it can be seen that for the short channel device $(\mathrm{L}=30 \mathrm{~nm})$, thermal noise current shows quite different trends for the cases where self-heating is activated and inactivated. For low $\mathrm{V}_{\mathrm{DS}}$ values, the two curves are almost overlapping. On the other hand, as $V_{D S}$ increases, the curves start to split. This is because of the higher power dissipation under larger $\mathrm{V}_{\mathrm{DS}}$ and the consequent higher operating temperature due to 
TABLE I: Parameters for the devices with the gate lengths of $30 \mathrm{~nm}$ and $300 \mathrm{~nm}$.

\begin{tabular}{lccccc}
\hline Parameter & $\mathrm{L}$ & $\mathrm{W}$ & Power & $\left.g_{m}\right|_{\mathrm{V}_{\mathrm{DS}}=1 V}$ & $g_{d 0}$ \\
\hline Unit & $\mathrm{nm}$ & $\mathrm{nm}$ & $\mathrm{mW}$ & $\mathrm{mA} / \mathrm{V}$ & $\mathrm{mA} / \mathrm{V}$ \\
\hline Short Channel & 30 & $20 \times 305$ & 6.25 & 9.14 & 20.01 \\
Long Channel & 300 & $20 \times 1068$ & 5.86 & 17.66 & 20.00 \\
\hline
\end{tabular}

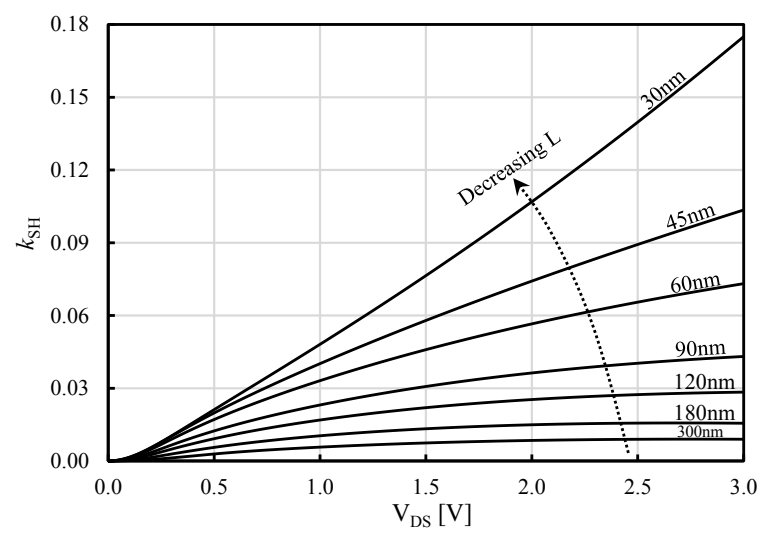

Fig. 3: Self-heating error factor $\left(k_{\mathrm{SH}}\right)$ with respect to $\mathrm{V}_{\mathrm{DS}}$ for devices with different gate lengths from $30 \mathrm{~nm}$ to $300 \mathrm{~nm}$.

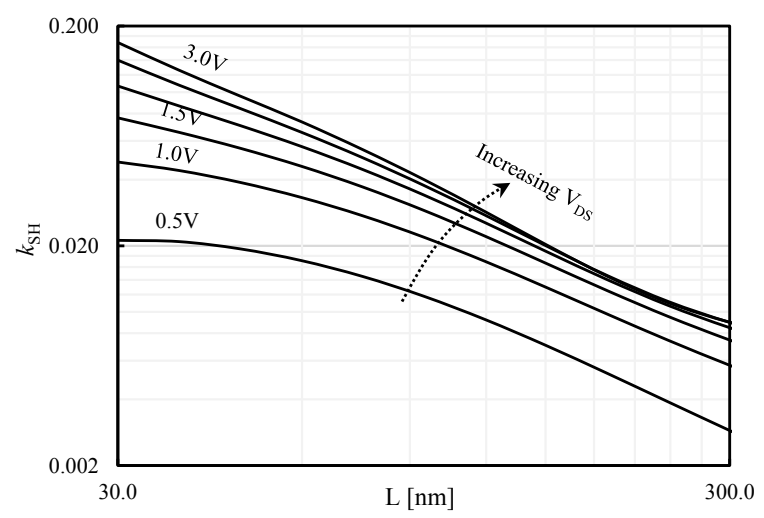

Fig. 4: Self-heating error factor $\left(k_{\mathrm{SH}}\right)$ with respect to gate length for $\mathrm{V}_{\mathrm{DS}}=0.5 \mathrm{~V}, 1.0 \mathrm{~V}, 1.5 \mathrm{~V}, 2.0 \mathrm{~V}, 2.5 \mathrm{~V}, 3.0 \mathrm{~V}$. For $\mathrm{V}_{\mathrm{DS}}=0 \mathrm{~V}, k_{\mathrm{SH}}=0$.

self-heating. The same observation can also be made for the long channel device $(\mathrm{L}=300 \mathrm{~nm})$; however, the difference is much less significant than the short channel device. This is not due to the lower power dissipation of the long channel device when compared to the short channel one. In fact, their power dissipation values are more or less the same (Table I); however, the short channel device has a much smaller area and its resulting power density is much larger. Therefore, the temperature of the short channel device rises to a much higher value due to its self-heating and that results in a large thermal noise current. For observing the effect of self-heating on the thermal noise current, we can define the self-heating error factor $k_{\mathrm{SH}}$ as

$$
k_{\mathrm{SH}}=\frac{S_{i, \mathrm{SH}} \quad S_{i}}{S_{i}}
$$

where $S_{i, \mathrm{SH}}$ is the thermal noise current (black curves on Fig. 2 ) when self-heating is considered and $k_{\mathrm{SH}}$ is the self-heating

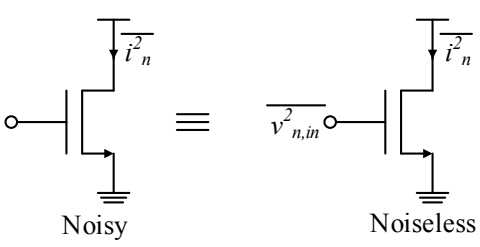

Fig. 5: Model of the noisy device with a fictitious input referred noise voltage source $\overline{v_{n, i n}^{2}}$

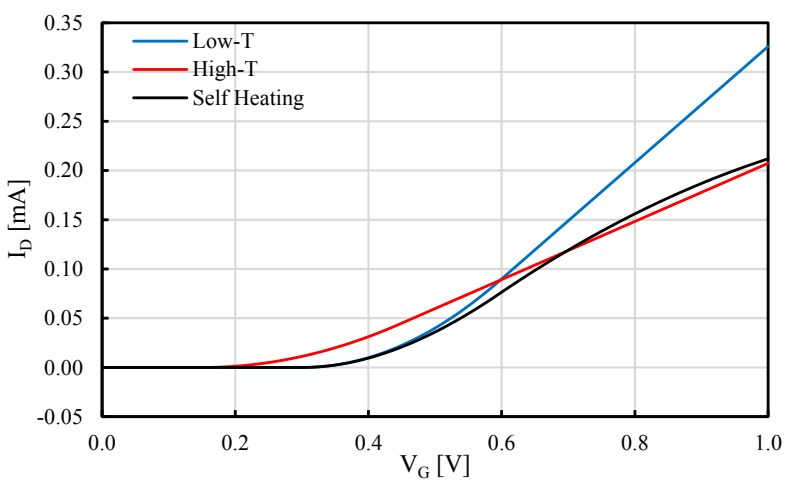

Fig. 6: $V_{G}-I_{D}$ curves for low temperature, high temperature and activated self-heating. The curves are for illustration of the behaviour of $g_{m}$ under different thermal conditions.

error factor. Therefore, by combining (1) and (3) $S_{i, \mathrm{SH}}$ can be written as

$$
S_{i, \mathrm{SH}}=4 k T\left(1+k_{\mathrm{SH}}\right) \gamma g_{d 0}=\left(1+k_{\mathrm{SH}}\right) S_{i} .
$$

Fig. 3 and Fig. 4 show the amount of error when self-heating is not taken into account. The error becomes larger linearly with increasing $\mathrm{V}_{\mathrm{DS}}$ and exponentially with decreasing gate length. Moreover, the error can go up to $18 \%$ under very large $\mathrm{V}_{\mathrm{DS}}$ for $30 \mathrm{~nm}$ gate length.

\section{Input Referred Thermal Noise Voltage}

In many applications like LNA design, the figure of merit for noise performance is the voltage quantity instead of the current since a voltage level is sensed at the output of the previous stage rather than the current. Hence, the performance of blocks are measured by parameters like input referred noise voltage or noise figure. Therefore, one should consider the self-heating effects also on the other parameters that influence the input referred noise voltage. The input referred noise voltage (Fig. 5) of a device can be expressed as (Fig. 5)

$$
S_{v, i n}=\frac{S_{i}}{g_{m}^{2}}=\frac{4 k T \gamma g_{d 0}}{g_{m}^{2}}
$$

where $g_{m}$ is the gate-to-drain transconductance of the device. In (5), $g_{d 0}$ is not influenced by the self-heating since the parameter is set at the operating point where $V_{D S}$ and the power dissipation is zero. However, $g_{m}$ has a dependency on the temperature, therefore it will be influenced by self- 
heating. Changing the temperature modifies $g_{m}$ mainly due to the temperature dependency of two other parameters that are the threshold voltage $\left(\mathrm{V}_{\mathrm{T}}\right)$ and the mobility $(\mu)$ [7]. Since $g_{m}$ is the first derivative of the drain current $\mathrm{I}_{\mathrm{D}}$, we can analyse its temperature dependence by observing the $\mathrm{V}_{\mathrm{G}}-\mathrm{I}_{\mathrm{D}}$ curves for different temperatures (Fig. 6). At higher temperatures, $\mathrm{V}_{\mathrm{T}}$ decreases. With a smaller $\mathrm{V}_{\mathrm{T}}$, the device turns on at a lower $\mathrm{V}_{\mathrm{G}}$, which means that for moderate-to strong inversion saturation, $\mathrm{I}_{\mathrm{D}}$ would increase more quickly at higher temperatures; hence $g_{m}$ would increase with temperature. On the other hand, $\mu$ gets smaller at larger temperatures. Therefore, once the device is turned on, the rate of change of $I_{D}$ would be lower at higher temperatures. As a result of this, for devices in strong inversion and in saturation, $g_{m}$ would increase with increasing temperature. Considering these two opposite trends, with the increasing temperature, $g_{m}$ would be larger for lower $\mathrm{V}_{\mathrm{G}}$ values and it starts to be smaller for higher $\mathrm{V}_{\mathrm{G}}$. Since the temperature increase due to device self-heating is more prominent for large $\mathrm{V}_{\mathrm{G}}$ (strong inversion and saturation), we assume that self-heating decreases $g_{m}$ inside the region of interest. Consequently, input referred thermal noise voltage increases according to (5) which worsens the noise figure of an amplifier.

Fig. 7 shows the input referred thermal noise voltage for the two cases where self-heating thermal model is active and inactive. It can be seen that under larger power dissipation, the difference between the two cases becomes also larger for the input referred noise voltage. For large $V_{D S}$ values, the error can be more than $35 \%$, which is twice larger than the error made in the thermal noise current. This is because $g_{m}$ also drops with self-heating. Moreover, the optimum biasing point, where $S_{v, \text { in }}$ is minimum, shifts slightly down when selfheating is considered. Consequently, one might miscalculate the optimum bias point, if self-heating effect is not taken into account. It can also be observed that the noise increases as the device is deeper in saturation. Therefore, for having the lowest possible noise voltage, the devices should be biased closer to the linear region so that the undesired effects, like excessive self-heating, hot electrons, high velocity saturation and avalanche multiplication, are prevented as much as possible.

\section{CONCLUSION}

In this paper, the thermal noise performance of MOSFET devices in 28nm FD-SOI technology is examined by considering their self-heating effects with a compact selfheating thermal model. It is shown that the thermal noise current $\left(S_{i}\right)$ of short channel devices increases significantly with self-heating of the device. For large $\mathrm{V}_{\mathrm{DS}}$ values, the increase can be around $18 \%$. In addition to this, the input referred thermal noise voltage $\left(S_{n, i n}\right)$ also increases due to self-heating. Besides, the increase in $S_{n, i n}$, which can go up to $37 \%$ at large $\mathrm{V}_{\mathrm{DS}}$ values, is even more prominent than $S_{i}$ mainly due to the drop in $g_{m}$ at larger temperature values because of self-heating.

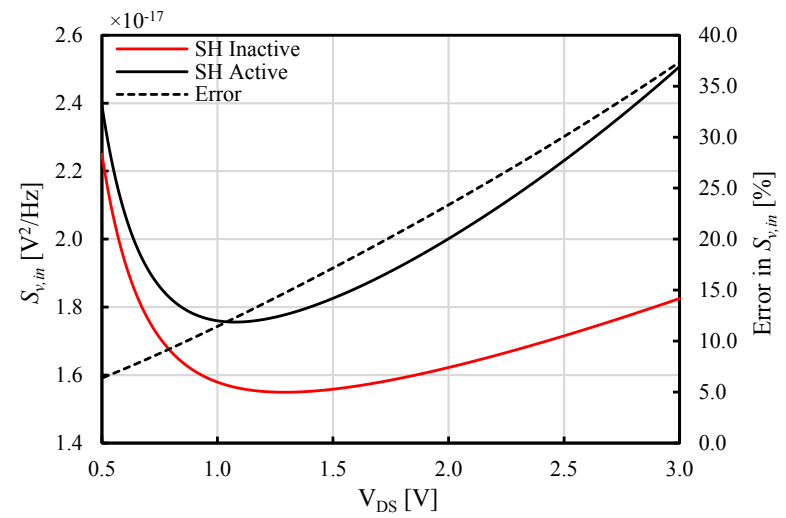

Fig. 7: Input referred thermal noise voltage $\left(S_{v, i n}\right)$ of $30 \mathrm{~nm}$ device for self-heating thermal model active and inactive and the percentage of error.

\section{REFERENCES}

[1] M. Pedram and S. Nazarian, "Thermal modeling, analysis, and management in vlsi circuits: Principles and methods," Proceedings of the IEEE, vol. 94, no. 8, pp. 1487-1501, Aug 2006.

[2] E. Pop, S. Sinha, and K. E. Goodson, "Heat generation and transport in nanometer-scale transistors," Proceedings of the IEEE, vol. 94, no. 8, pp. 1587-1601, Aug 2006.

[3] K. Raleva, D. Vasileska, A. Hossain, S.-K. Yoo, and S. M. Goodnick, "Study of self-heating effects in soi and conventional mosfets with electro-thermal particle-based device simulator," Journal of Computational Electronics, vol. 11, no. 1, pp. 106-117, 2012.

[4] M. Mohamed, Z. Aksamija, W. Vitale, F. Hassan, K.-H. Park, and U. Ravaioli, "A conjoined electron and thermal transport study of thermal degradation induced during normal operation of multigate transistors," IEEE Transactions on Electron Devices, vol. 61, no. 4, pp. 976-983, April 2014.

[5] C. Baltaci and Y. Leblebici, "Thermal issues in deep sub-micron fdsoi circuits," in 2016 12th Conference on Ph.D. Research in Microelectronics and Electronics (PRIME), June 2016, pp. 1-4.

[6] A. Van der Ziel, Noise; sources, characterization, measurement, ser. Prentice-Hall information and system sciences series. Prentice-Hall, 1970.

[7] Y. Tsividis, Operation and Modeling of the MOS Transistor. New York, NY, USA: McGraw-Hill, Inc., 1987.

[8] P. Klein, "An analytical thermal noise model of deep submicron mosfet's," IEEE Electron Device Letters, vol. 20, no. 8, pp. 399-401, Aug 1999.

[9] A. J. Scholten, H. J. Tromp, L. F. Tiemeijer, R. V. Langevelde, R. J. Havens, P. W. H. D. Vreede, R. F. M. Roes, P. H. Woerlee, A. H. Montree, and D. B. M. Klaassen, "Accurate thermal noise model for deep-submicron cmos," in International Electron Devices Meeting 1999. Technical Digest (Cat. No.99CH36318), Dec 1999, pp. 155-158.

[10] G. Knoblinger, P. Klein, and M. Tiebout, "A new model for thermal channel noise of deep submicron mosfets and its application in rfcmos design," in 2000 Symposium on VLSI Circuits. Digest of Technical Papers (Cat. No.00CH37103), June 2000, pp. 150-153.

[11] R. P. Jindal, "Hot-electron effects on channel thermal noise in fine-line nmos field-effect transistors," IEEE Transactions on Electron Devices, vol. 33, no. 9, pp. 1395-1397, Sep 1986.

[12] A. A. Abidi, "High-frequency noise measurements on fet's with small dimensions," IEEE Transactions on Electron Devices, vol. 33, no. 11, pp. 1801-1805, Nov 1986.

[13] A. Van Der Ziel, Noise in Solid State Devices and Circuits. Wiley, 1986.

[14] A. J. Scholten, L. F. Tiemeijer, R. van Langevelde, R. J. Havens, A. T. A. Z. van Duijnhoven, and V. C. Venezia, "Noise modeling for $\mathrm{rf} \mathrm{cmos}$ circuit simulation," IEEE Transactions on Electron Devices, vol. 50, no. 3, pp. 618-632, March 2003.

[15] R. O, M. A. Jaud, T. Poiroux, and M. Benosman, UTSOI Model 1.1.4: Surface Potetntial Model for Ultra Thin Fully Depleted SOI MOSFET, LETI: Laboratoire d'électronique et de technologie de l'information, November 2012. 